\title{
Genome-wide identification of HSF Family in Peach and Functional analysis of PpHSF5 involvement in Root and Aerial Organ Development
}

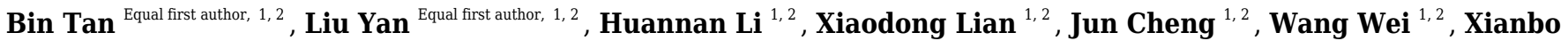 \\ Zheng $^{1,2}$, Xiaobei Wang ${ }^{1,2}$, Jidong Li $^{1,2}$, Xia Ye ${ }^{1,2}$, Langlang Zhang ${ }^{1,2}$, Zhiqian Li ${ }^{1,2}$, Jiancan Feng ${ }^{\text {Corresp. } 1,2}$ \\ ${ }^{1}$ College of Horticulture, Henan Agricultural University, Zhengzhou, China \\ Henan Key Laboratory of Fruit and Cucurbit Biology, Zhengzhou, China \\ Corresponding Author: Jiancan Feng \\ Email address: jcfeng@henau.edu.cn
}

Background. Heat shock factors (HSFs) play important roles during normal plant growth and development and when plants respond to diverse stressors. Although most studies have focused on the involvement of HSFs in the response to abiotic stresses, especially in model plants, there is little research on their participation in plant growth and development or on the HSF (PpHSF) gene family in peach (Prunus persica).

Methods. DBD (PF00447), the HSF characteristic domain, was used to search the peach genome and identify PpHSFs. Phylogenetic, multiple alignment and motif analyses were conducted using MEGA 6.0, ClustalW and MEME, respectively. The function of PpHSF5 was confirmed by overexpression of PpHSF5 into Arabidopsis.

Results. Eighteen PpHSF genes were identified within the peach genome. The PpHSF genes were nonuniformly distributed on the peach chromosomes. Seventeen of the PpHSFs (94.4\%) contained one or two introns, except PpHSF18, which contained three introns. The in silico-translated PpHSFs were classified into three classes (PpHSFA, PpHSFB and PpHSFC) based on multiple alignment, motif analysis and phylogenetic comparison with HSFs from Arabidopsis thaliana and Oryza sativa. Dispersed gene duplication (DSD at 67\%) mainly contributed to HSF gene family expansion in peach. Promoter analysis showed that the most common cis-elements were the MYB (abiotic stress response), ABRE (ABAresponsive) and MYC (dehydration-responsive) elements. Transcript profiling of 18 PpHSFs showed that the expression trend of PpHSF5 was consistent with shoot length changes in the cultivar 'Zhongyoutao 14'. Further analysis of the PpHSF5 was conducted in 5-year-old peach trees, Nicotiana benthamiana and Arabidopsis thaliana, respectively. Tissue-specific expression analysis showed that PpHSF5 was expressed predominantly in young vegetative organs (leaf and apex). Subcellular localization revealed that PpHSF5 was located in the nucleus in N. benthamiana cells. Two transgenic Arabidopsis lines were obtained that overexpressed PpHSF5. The root length and the number of lateral roots in the transgenic seedlings were significantly less than in WT seedlings and after cultivation for three weeks. The transgenic rosettes were smaller than those of the WT at 2-3 weeks. The two transgenic lines exhibited a dwarf phenotype three weeks after transplanting, although there was no significant difference in the number of internodes. Moreover, the PpHSF5-OE lines exhibited enhanced thermotolerance. These results indicated that PpHSF5 might be act as a suppresser of growth and development of root and aerial organs. 
1 Genome-wide Identification of HSF Family in Peach and

2 Functional Analysis of PpHSF5 involvement in Root and

3 Aerial Organ Development

4

5 Bin Tan ${ }^{1,2 *}$, Liu Yan ${ }^{1,2 *}$, Huannan $\mathrm{Li}^{1,2}$, Xiaodong Lian ${ }^{1,2}$, Jun Cheng ${ }^{1,2}$, Wei Wang ${ }^{1,2}$, Xianbo

6 Zheng ${ }^{1,2}$, Xiaobei Wang ${ }^{1,2}$, Jidong $\mathrm{Li}^{1,2}$, Xia Ye ${ }^{1,2}$, Langlang Zhang ${ }^{1,2}$, Zhiqian $\mathrm{Li}^{1,2}$ and Jiancan

$7 \quad$ Feng 1,2

8

$9{ }^{1}$ College of Horticulture, Henan Agricultural University, Zhengzhou 450002, China;

$10{ }^{2}$ Henan Key Laboratory of Fruit and Cucurbit Biology, Zhengzhou 450002, China

$11 *$ These authors contributed equally to this work.

12 Corresponding Author:

13 Jiancan Feng ${ }^{1,2}$

14 Nongye Road No. 63, Zhengzhou, Henan Province, 450002, China

15 Email address: jcfeng@,henau.edu.cn 
16 Abstract

17 Background. Heat shock factors (HSFs) play important roles during normal plant growth and development and when plants respond to diverse stressors. Although most studies have focused on the involvement of HSFs in the response to abiotic stresses, especially in model plants, there is little research on their participation in plant growth and development or on the HSF (PpHSF) gene family in peach (Prunus persica).

Methods. DBD (PF00447), the HSF characteristic domain, was used to search the peach genome and identify PpHSFs. Phylogenetic, multiple alignment and motif analyses were conducted using MEGA 6.0, ClustalW and MEME, respectively. The function of PpHSF5 was confirmed by overexpression of PpHSF5 into Arabidopsis.

Results. Eighteen $P p H S F$ genes were identified within the peach genome. The PpHSF genes were nonuniformly distributed on the peach chromosomes. Seventeen of the PpHSFs (94.4\%) contained one or two introns, except PpHSF18, which contained three introns. The in silicotranslated PpHSFs were classified into three classes (PpHSFA, PpHSFB and PpHSFC) based on multiple alignment, motif analysis and phylogenetic comparison with HSFs from Arabidopsis thaliana and Oryza sativa. Dispersed gene duplication (DSD at 67\%) mainly contributed to HSF gene family expansion in peach. Promoter analysis showed that the most common cis-elements were the MYB (abiotic stress response), ABRE (ABA-responsive) and MYC (dehydrationresponsive) elements. Transcript profiling of 18 PpHSFs showed that the expression trend of PpHSF5 was consistent with shoot length changes in the cultivar 'Zhongyoutao 14'. Further 
36 analysis of the PpHSF5 was conducted in 5-year-old peach trees, Nicotiana benthamiana and

37 Arabidopsis thaliana, respectively. Tissue-specific expression analysis showed that PpHSF5 was

38 expressed predominantly in young vegetative organs (leaf and apex). Subcellular localization

39 revealed that PpHSF5 was located in the nucleus in N. benthamiana cells. Two transgenic

40 Arabidopsis lines were obtained that overexpressed PpHSF5. The root length and the number of

41 lateral roots in the transgenic seedlings were significantly less than in WT seedlings and after

42 cultivation for three weeks. The transgenic rosettes were smaller than those of the WT at 2-3

43 weeks. The two transgenic lines exhibited a dwarf phenotype three weeks after transplanting,

44 although there was no significant difference in the number of internodes. Moreover, the

45 PpHSF5-OE lines exhibited enhanced thermotolerance. These results indicated that PpHSF5

46 might be act as a suppresser of growth and development of root and aerial organs.

48 Key Words Heat shock factors family, Root development, Peach (Prunus persica), Aerial organ, 49 Functional identification, PpHSF5 


\section{Introduction}

51 Plant growth and development are affected by a range of abiotic stress, including cold, heat,

52 salinity and drought stress (Guo et al., 2016). Heat shock factors (HSFs) act with heat shock

53 proteins (HSPs) as key transcriptional activators during responses to abiotic stress ( Hu et al.,

54 2009). Recent studies indicated that HSFs act as key components of signal transduction in

55 response to different abiotic stresses in plants (Guo et al., 2016; Scharf et al., 2012).

56 HSFs in plant genomes can be identified by a conserved DNA-binding domain (DBD). The

57 DBD domain is located in the N-terminal of all HSFs and specifically binds to heat stress (HS)

58 motifs in the promoters of target genes (Wang et al., 2018). The adjacent HR-A/B region is

59 linked to the DBD by a connector of variable length (15-80 amino acid residues) that contains a

60 bipartite heptad pattern of hydrophobic amino acid residues, which constitutes a coiled-coil

61 domain for protein interaction. According to the number of amino acid residues inserted into the

62 HR-A/B region, HSFs are divided into three main groups, each with subgroups, namely HSFA

63 (A1-A9), HSFB (B1-B5) and HSFC (C1-C2) (Koskull-Doring, Scharf \&Nover, 2007; Yang et al.,

64 2014). HSFA members contain an acidic motif (AHA activation domain) at their C-terminus and

65 act as transcriptional activators. The members of HSFB as transcriptional repressors.

66 In a wide range of plants, a number of HSFs have been shown to be involved in resistance to

67 heat (Guo et al., 2016) and other abiotic or biotic stresses (Yu et al., 2019). Of the 21 HSF family

68 members in Arabidopsis, a number act as pioneer regulators of the response to heat shock.

69 HSFA1a, HSFA1b, HSFA1d, HSFA1e and HSFA2 play active regulatory roles in the response

Peer] reviewing PDF | (2020:02:45572:5:0:NEW 10 Jan 2021) 
70 to HS in plants (Busch et al., 2005; Nishizawa et al., 2006). In Arabidopsis, the assembly of the

71 HSFA1/A2 super-activated complex regulates heat stress (HS) genes (Chan-Schaminet et al.,

72 2009). $H S F B 1$ and $H S F B 2 b$ participate in disease resistance regulation of Arabidopsis and

73 expression of Pdf1.2 (Kumar et al., 2009). OsHSFB4b and OsHSFA2c participate in the

74 regulation of the heat shock response by regulating the expression of HSP100 (Singh et al.,

75 2012). OsHSFC1b is related to the regulation of salt stress and plant development (Schmidt et al.,

76 2012).

77 Several HSFs are stress-inducible transcriptional factors that participate in the growth and

78 development of root and aerial organs in plant. Overexpression of AtHsfB4 in Arabidopsis

79 induces specific effects on root development, resulting in shortened roots (Begum et al., 2013).

80 The over-expression of $B h H s f l$ conferred growth retardation of aerial organs, producing a dwarf

81 phenotype, although the primary roots were not obviously different from those of wild type (Zhu

82 et al., 2009). Transgenic Arabidopsis plants with strong expression of AtHsfA3 and AtHsfA2

83 showed a severely dwarfed phenotype and increased tolerance to heat (Ogawa et al., 2007;

84 Yoshida et al., 2008). The thermotolerant phenotype was also observed in the cotyledons, rosette

85 leaves, inflorescence stems and seeds of transgenic Arabidopsis plants expressing OsHsfA2e

86 (Yokotani et al., 2008).

87 The HSF family have been analyzed genome-wide in several plants, such as rice (Oryza sativa), Arabidopsis (Arabidopsis thaliana), cotton (Gossypium hirsutum), soybean (Glycine max), wheat (Triticum aestivum), pepper (Capsicum annuum), poplar (Populus trichocarpa), 
90

91

92

93

94

95

Brassica napus, grape (Vitis vinifera) and Tartary buckwheat (Fagopyrum tataricum) ( Nover et al., 2001; Chauhan et al., 2011; Wang et al., 2014; Li et al., 2014; Guo et al., 2015; Xue et al., 2014; Zhang et al., 2016; Zhu et al., 2017; Liu et al.,2018; Liu et al., 2019). Peach (Prunus persica L.) is an important economical crop and a popular fruit with consumers. However, there are limited studies on peach HSFs. To remedy this, we identified the HSF family in the peach genome and conducted bioinformatics analysis of the 18 identified PpHSFs. Based on the latest transcriptome data (Lian et al., 2020), the expression patterns of the PpHSF genes were analyzed during development of the cultivar 'Zhongyoutao 14'. 'Zhongyoutao 14' (derived from 'SD9238'), is a temperature-sensitive peach that exhibits a shorter internode length and a smaller canopy when grown below $30^{\circ} \mathrm{C}$ (Lu et al., 2016). PpHSF5 was further analyzed and found to function in the development of the root and aerial organs. Furthermore, the thermotolerant phenotype was analyzed in newly obtained transgenic Arabidopsis plants expressing PpHSF5.

The genome-wide analysis of PpHSF gene family offers a basis for further investigation into the function and evolutionary history of peach $H S F s$ and provides candidate genes for peach molecular breeding.

\section{Materials \& Methods}

\section{Plant Materials}

Established peach trees (Prunus persica) cultivar 'Zhongyoutao 14' ('Maotao' as rootstock) have 
109

110

111

112

113

114

115

116

117

118

119

120

121

122

123

124

125

126

127

been grown for 5 years at the Experimental Station of the Horticulture College, Henan

Agricultural University (Zhengzhou, China). Samples from the apex, young and mature leaves,

self-pollinated embryos, and fruit were collected, frozen in liquid nitrogen and stored at $-80^{\circ} \mathrm{C}$.

Leaves from Nicotiana benthamiana were used for subcellular location of PpHSF5. Arabidopsis

thaliana (L.) Heynh Columbia 0 (Col-0) was used for transformation with PpHSF5.

\section{Identification and Chromosomal Location of HSF Genes in Peach}

The hidden Markov model (HMM) of the DBD domain (PF00447), characteristic of HSFs, was downloaded from the Pfam website (Finn et al., 2016) and used to identify HSF genes in peach.

The peach genome files (v2.1) were downloaded from JGI database (https://phytozome.jgi.

doe.gov/pz/portal.html) (Verde et al., 2013), HSF protein sequences were obtained in peach genome by BLASTP and hmmsearch function, and then the DBD domain were further identified by Pfam analysis. The peach HSF gene and protein sequences were extracted from Phytozome v12.1. PpHSF genes were named according to physical location on the chromosomes. Positional information was retrieved from peach genome annotations obtained from Phytozome v12.1, and chromosome locations of the PpHSFs were drawn using the Circos software (Krzywinski et al., 2009). The isoelectric points and other physical properties were approximated from ExPASy (http://web.expasy.org/compute_pi). Gene structures were predicted using the Gene Structure Display Server 2.0 (http://gsds.cbi.pku.edu.cn/).

\section{Phylogenetic and Motif Analysis of PpHSFs}


128 The amino acid sequences of 21 AtHSFs (Arabidopsis thaliana), 25 OsHSFs (Oryza sativa) and

12918 PpHSFs (Prunus persica) were gathered from Phytozome v12.1 using ClustalW with system

130 default settings. The phylogenetic trees were formulated by the maximum likelihood method

131 (ML) with Jones-Taylor-Thornton (JTT) model in MEGA 6.0

132 (http://www.megasoftware.net/download_form).. Conserved motifs of HSF proteins in peach

133 were identified using the MEME tool (http://meme.nbcr.net/meme/cgi-bin/meme.cgi) with

134 default parameters in normal operation mode. The subcellular localization was predicted with

135 Plant-mPLoc (http://www.csbio.sjtu.edu.cn/bioinf/plant-multi/\#).

\section{Gene Duplication and Cis-Element Analysis of PpHSFs}

137 Gene duplication was analyzed using MCScanX (Wang et al., 2012). Genomic DNA sequences 138 (2000 bps upstream of the start codons) for each PpHSF were obtained from the peach genome and skimmed in the PlantCARE database

(http://bioinformatics.psb.ugent.be/webtools/plantcare/html/) for cis-acting elements analysis of

141 the promoter in PpHSFs.

\section{Gene Expression Analysis of PpHSFs}

143 The FPKM (fragments per kilobase of exon per million fragments mapped) values of the 18 shoots at four critical growth stages, namely initial period (IP), initial elongation period (IEP), rapid growth period (RGP) and stable growth period (SGP) of temperature-sensitive peach 
147 cultivar 'Zhongyoutao 14' (Lian et al., 2020). The average maximum temperature of previous

148 week (AMTPW) began to be higher than $30^{\circ} \mathrm{C}$ in the first day of RGP (Lian et al., 2020). The

149 heat map was generated by TBtools (Chen et al., 2020).

\section{Quantitative Real-time PCR Analysis of PpHSF5}

151 Total RNA of different tissues from 'Zhongyoutao 14' peach and leaves from $\mathrm{T}_{2}$ transgenic

152 Arabidopsis lines was isolated using the Spin Column Plant Total RNA Purification Kit

153 (ShengGong, Shanghai, China). The cDNA was synthesized using FastQuant RT Kit (Tiangen

154 Biotech, Beijing, China). qRT-PCR was implemented using an ABI PRISM 7500 FAST

155 Sequence Detection System (Applied Biosystems, Madrid, CA, USA) with SYBR Select Master

156 Mix (Applied Biosystems, USA). Primers of PpHSF5 were designed using Primer Premier 5.0.

$157 P p G A P D H$ (Prupe.1G234000) and $A t U B C$ (AT5G25760) were used as constitutive controls for

158 either tissue-specific expression in peach or expression analysis in transgenic Arabidopsis,

159 respectively. Primers are shown in Supplemental Table S1-2. The reaction mixture was as

160 follows: $1 \mu \mathrm{L}$ cDNA template $(200 \mathrm{ng} / \mu \mathrm{L}), 1 \mu \mathrm{L}$ of each primer $(10 \mu \mathrm{M}), 10 \mu \mathrm{L}$ SYBR Premix

161 and $7 \mu \mathrm{L} \mathrm{ddH_{2 }} \mathrm{O}$. Melting curve analysis was performed after the end of 40 cycles to insure

162 proper amplification of the target. During the melting process, fluorescence readings were

163 continuously collected from $60-90^{\circ} \mathrm{C}$ at a heating rate of $0.5^{\circ} \mathrm{C} \mathrm{s}^{-1}$. All analysis was repeated

164 three times using biologically replicates. The relative expression levels of PpHSF5 were

165 calculated as $2^{-\Delta \Delta \mathrm{CT}}$ method (Schmittgen et al., 2008). The relative expression levels of PpHSF5

166 was calculated in SPSS using ANOVA at significance levels of $P<0.05$.

Peer] reviewing PDF | (2020:02:45572:5:0:NEW 10 Jan 2021) 
167 Subcellular Localization of PpHSF5

168 PpHSF5 without the termination codon was amplified by PCR using cDNA from 'Zhongyoutao

169 14' as the template (Primer details in Supplemental Table S1-2). This coding region was cloned

170 into the pSAK277-GFP vector to construct PpHSF5::GFP fusion proteins that were driven by the

171 CaMV 35S promoter. The p35SPpHSF5::GFP and p35SGFP (control) vectors were transformed

172 into Agrobacterium tumefaciens strain GV3101, which were then injected into leaves of $N$.

173 benthamiana according to previously published protocols (Sparkes et al., 2006). The leaves were

174 observed 48-72 $\mathrm{h}$ after injection using laser scanning confocal microscopy (Zeiss LSM700).

175 Construction of Expression Vectors for Plant Transformation

176 The CDS of PpHSF5 was PCR-amplified and cloned into the pSAK277 vector using the

177 restriction enzymes Xho I and Xba I (Primer details in Supplemental Table S1-2). The

178 p35S::PpHSF5 vector was transformed into Agrobacterium tumefaciens strain GV3101. The

179 floral dip method was used to transform Arabidopsis thaliana (Col-0) (Chung et al., 2000).

180

181

182

183

184

185

\section{Phenotype of Overexpression PpHSF5 in Arabidopsis}

The seeds from $\mathrm{T}_{2}$ transgenic Arabidopsis lines were sterilized by $6.25 \% \mathrm{NaClO}$ for $5 \mathrm{~min}$, and then washed in sterilized $\mathrm{ddH}_{2} \mathrm{O}$. The seeds were cultured at $4^{\circ} \mathrm{C}$ for $2 \mathrm{~d}$ and then transferred onto MS solid medium under 16/8 h light/dark cycle for one week on square plates. Three biological replicates (with three seedlings of each lines per square plate) were used for observation of root phenotype. The roots of different transgenic lines with three plants per line 
186 187 188 189 190 191 192 193 194

were measured by a LA2400 Scanner at three weeks to determine the growth status. The leaves were cut from the transgenic seedlings for gene expression analysis. Other seedlings, germinated on agar and grown for one week, were transferred into the soil and grown under normal conditions. The length and width of rosettes (four leaves per plants, five repetition) and number of rosettes (five plants per line) in different transgenic lines and WT were measured and photographed at two weeks and at three weeks after transplanting, respectively. Moreover, the morphology of transgenic lines and WT, including the height of plants (eight plants per line) and the number of branches and blooms (five plants per line) were recorded, three weeks after transplanting.

\section{Heat Stress Treatment}

For performing heat stress treatment on the seeds germination and plants grown on the agar medium, seeds of WT and transgenic Arabidopsis lines sown on MS medium at $4^{\circ} \mathrm{C}$ for $2 \mathrm{~d}$ and in darkness for $2 \mathrm{~d}\left(22^{\circ} \mathrm{C}\right)$ were exposed to HS stress at $46^{\circ} \mathrm{C}$ for $30 \mathrm{~min}$, and then were transferred into a climate chamber $\left(22^{\circ} \mathrm{C}, 16 \mathrm{~h}\right.$ light $/ 8 \mathrm{~h}$ dark cycles $)$. After HS treatment, the germination of seeds were counted daily and photographed. More than 50 seeds of each line were used in each plate with three replications. Difference in HS stress was confirmed using $t$ test.

\section{Statistical Analysis}

Data were analyzed by ANOVA, Tukey $\mathrm{HSD}^{\mathrm{a}}$ and Duncan ${ }^{\mathrm{a}}$ 's multiple range tests $($ at $P<0.05)$ 
using IBM SPSS Statistics 20 (SPSS, USA).

206

207

\section{Results}

208

209

210

211

212

213

214

215

216

217

218

219

220

\section{Genome-wide Identification, Chromosomal Distribution and Gene Structures of HSF}

\section{Genes in Peach}

Eighteen HSF gene family members were identified from the peach genome and then named PpHSF1 to PpHSF 18 according to their physical locations (Table 1 and Supplemental Table S21). The PpHSF coding sequences ranged from 591 bp (PpHSF8) to 1608 bp (PpHSF14). In silico-translated PpHSF proteins showed divergent lengths [196 to 535 amino acids (aa)] with different molecular weights (22.36 59.56 kDa) and isoelectric points (4.67 to 8.75) (Table1). All PpHSFs were predicted to be nuclear-localized proteins.

Seven of the 8 peach chromosomes contained at least one $P p H S F$, with the exception being chromosome 6 (Fig. 1 and Table 1). Five PpHSFs were located on chromosome 1 (PpHSF1-5), and another five (PpHSF 13-17) on chromosome 7. Chromosomes 2, 3 and 8 carried only one PpHSF gene each, while chromosome 5 had two, and chromosome 4 had three. The above results indicated that PpHSFs were unevenly distributed across the peach chromosomes.

The structural differences of the PpHSF genes were also analyzed. The number of introns ranged from one to three among the PpHSFs. The majority of the PpHSFs $(66.67 \%)$ contained one intron, $27.78 \%$ contained two introns, and only PpHSF18 contained three introns 
224 (Supplemental Figure S1 and Supplemental Table S2-2). Interestingly, both PpHSF18 and

225 PpHSF12 has predicted introns in the 5'-UTR and 3'-UTR, respectively.

\section{Gene Duplication Pattern Analysis of PpHSFs}

227 To explain the expansion of the PpHSFs gene family, the gene duplication patterns of the

PpHSFs were analyzed and compared across the peach genome (Supplemental Table S3). There were only two patterns of gene duplications, with $67 \%$ of the gene pairs derived from dispersed gene duplication (DSD) and the remaining gene pairs derived from whole-genome duplication

(WGD). Three syntenic pairs were identified, and all originated from WGD. The syntenic genes were located on different chromosomes from their partner (Fig. 1).

\section{Classification, Phylogenetic and Motif Analyses of PpHSFs}

234

Among plant species, there are two characteristic amino acid domains in the HSF family, the DBD and adjacent HR-A/B region (Nover et al., 2001). The PpHSFs were divided into three classes (PpHSFA, PpHSFB and PpHSFC), according to the number of amino acids between part A and part B of the HR-A/B domain (Fig. 2A). Multiple sequence alignment analysis of the PpHSF proteins indicated that an insertion of 21 amino acids was found in Class A (11 of the PpHSFs) and a shorter insertion of 14 amino acid in Class C ( 1 of the PpHSFs) between the HRA and HR-B regions. Six of the PpHSFs had no aa insertion between the two domains (Class B). 
243

244

245

246

247

248

249

250

251

252

253

254

255

256

257

258

259

260

261

262

conducted by constructing a phylogenetic tree. According to the phylogenetic tree, the 64 HSFs derived from the three plant species were divided into three classes and 15 subclasses (Fig. 2B). The peach proteins sorted into the classes of HSFs, within (11 members) in class HSFA, six in HSFB, and one in HSFC. Class A included nine subclasses (A1-A9), the largest number of subclasses. The PpHSFs were grouped into eight of the Class A subclasses, with no PpHSF in Class A7. Class B consisted of 18 total members and was divided into four subclasses (B1-B4). It is noteworthy that PpHSF8 clustered with Class B but as a single branch. Only six members were clustered into Class $\mathrm{C}$, with two subclasses (C1-C2). No PpHSFs clustered with subclass $\mathrm{C} 2$.

The conserved motifs in the PpHSF proteins were analyzed using MEME. The results revealed that PpHSFs contained ten conserved motifs (Fig. 2C and Supplemental Table S4). Motifs 1-3 were found in the N-terminals (the most conserved region) of each PpHSF. Motif 4 was found in Class A and Class B. Motif 5, which was found between the HR-A and HR-B regions, was observed in Class A and Class C. The motif analysis was consistent with the multiple sequence alignment and phylogenetic analyses.

\section{Analysis of the Cis-Acting Regulatory Elements in the PpHSF Gene Promoters}

The cis-acting elements within the promoters of the 18 PpHSFs were analyzed using PlantCARE. Every promoter contained at least two MYB elements (abiotic stress response) (Table 2). All but one promoter contained an ABRE (ABA-responsive element). The next most common elements were MYC elements (dehydration-responsive) (in $88.8 \%$ of the promoters), CGTCA- and 
263 TGACG- motifs (83.3\%), and ARE elements (anaerobic induction) (77.8\%). ERE (ethylene-

264 responsive element), MBS (drought inducible), MRE and P-box elements were also present in 265 the promoters of some PpHSFs. The TCA-motif was observed in only five PpHSFs, namely

266 PpHSF1, PpHSF2, PpHSF5, PpHSF6 and PpHSF13. Previous studies reported several elements,

267 including MYB, ABRE, MYC, play vital roles in stress responses in plants (He et al., 2012; Li et

268 al., 2012). The different cis-elements in the promoter regions of these PpHSFs implied that the

269 PpHSFs may function in plant development and stress responses.

\section{The Expression Patterns of PpHSFs during shoot elongation in 'Zhongyoutao 14'}

271 Based on our previous RNA-seq analysis (Lian et al., 2020), the expression patterns of PpHSFS

272 were compared in four critical stages of shoot elongation of 'Zhongyoutao 14' grown under

273 elevated temperature in the field (Fig. 3). Most of PpHSFs belonging to the A and C classes

274 (except PpHSF4 and PpHSF11) were maintained at lower expression level. The PpHSFS of B

275 class exhibit diverse expression patterns. The FPKM values of $P p H S F 8$ and $P p H S F 13$ remained

276 almost unchanged at the four stages. The transcripts of another three PpHSFs (PpHSF15,

277 PpHSF6 and PpHSF12) were present at lower levels during the IEP stage and then slightly

278 increased during the RGP and SGP stages. The expression of level of PpHSF5 showed higher in

279 IEP stage and increased from the RGP to SGP stages. PpHSF5 might participate in temperature-

280 induced shoot growth of temperature-sensitive peach.

281 Expression Analysis of PpHSF5 and Subcellular Localization of PpHSF5

Peer] reviewing PDF | (2020:02:45572:5:0:NEW 10 Jan 2021) 
282 The relative expression of PpHSF5 was investigated by qRT-PCR in different organs of

283 'Zhongyoutao 14' (Fig. 4; supplement Table S5-1). The results showed that PpHSF5 were

284 expressed predominantly in young vegetative organs (leaves and apex), but barely detectable in

285 embryos and mature leaves. This suggested that PpHSF5 might participated in the growth and

286 development of plants. The 35S::PpHSF5-GFP signal was evident in the cellular nucleus in $N$.

287 benthamiana cells, indicating a nuclear localization (Fig. 5). The result was in concurrence with

288 the prediction from Plant-mPLoc of subcellular localization (Table 1).

\section{Overexpression of PpHSF5 in Arabidopsis Results in Dwarf Phenotypes}

To investigate the function of PpHSF5, an overexpression vector with PpHSF5 was transformed into Arabidopsis. The phenotype of two transgenic lines and WT were recorded (Fig. 6). One week after germination on agar medium, the transgenic lines had shorter roots and a smaller number of lateral roots than WT seedlings (Fig. 6A). The average root length in WT was $7.13 \mathrm{~cm}$, in transgenic line L1 was $3.08 \mathrm{~cm}$, and in L2 was $3.50 \mathrm{~cm}$ (Fig. 6A and B; Supplemental Table S6-1). Two weeks after transplantation, there was no difference in the number of rosette leaves between the transgenic lines and WT (Fig. 6C and D-a and Supplemental Table S6-2), although the rosette leaves were significantly shorter and narrower in the transgenic lines (the average length and width; Fig. 6D-b, c and Supplemental Tables S6-3 and S6-4). The mRNA levels in the PpHSF5-OE lines were obviously higher than WT plants (Fig. 6D-d and Supplemental Table S5-2). 
302 the leaves were shorter and narrower than those in WT plants (Fig. 6F). Moreover, the two

303

304

305

306

307 transgenic lines (L1 and L2) exhibited a dwarf phenotype (Fig. 6E and F). The average height of L1 $(16.83 \mathrm{~cm})$ was $40 \%$ shorter than that of the WT $(26.77 \mathrm{~cm})$. The number of rosette branches was much greater in WT than in transgenic lines, which had just one flowering stalk (Fig. 6F).

There was no significant difference in the number of internodes (Fig. 6E and F), indicating that the dwarf phenotype of the transgenic lines might be caused by shorter internode length.

transplanting (Fig. 6G and H). Root length and root volume were significantly lower in transgenic lines compared to WT (Fig. 6G and H, Supplemental Table S6-12, 13). The average length of roots in Line 1 was $219.34 \mathrm{~cm}$, which was $54 \%$ of the length in WT plants. The root volumes in the transgenic lines (Line 1 was $0.19 \mathrm{~cm}^{3}$, Line 2 was $0.36 \mathrm{~cm}^{3}$ ) was no more than $20 \%$ of that in WT $\left(1.95 \mathrm{~cm}^{3}\right)$. Other root indexes output by the root scanner were also less in the PpHSF5-OE lines, including the forks, tips and crossings of roots (Fig. 6G and H, Supplemental Table S6-14, 15, 16). Between the two transgenic lines, the higher expression level of PpHSF5 in L1 resulted in more obvious phenotypes compared to PpHSF5-OE L2 and WT (Fig. 6A, C, and E and Supplemental Table S5-2). The above results indicated that PpHSF5 might participate in plant growth and development and that overexpression of PpHSF5 results in a dwarf phenotype in transgenic Arabidopsis.

\section{PpHSF5-OE Lines Exhibit Enhanced Thermotolerance}

The thermotolerance of PpHSF5-OE lines was assayed with that of WT (Fig 7 and 
322 Supplemental Table S7). As shown in Fig 7B and F, only 8.3\% WT seeds germinated, whereas

323 more than $93.3 \%$ of the transgenic seeds germinated after HS treatment $1 \mathrm{~d}$. Nearly half of the

324 WT seeds germinated after HS treatment $3 \mathrm{~d}$, whereas $100 \%$ of the transgenic seeds were

325 germinated (Fig 7C and F). After HS treatment $5 \mathrm{~d}$ and $7 \mathrm{~d}, 68.4 \%$ and $82.6 \%$ of WT seeds

326 germinated, respectively (Fig 7D, E and F). Compared to WT seedlings, the PpHSF5-OE

327 seedlings exhibited green cotyledons and vigor growth (Fig 7C, D and E). These results suggested that the overexpression of PpHSF5 improves thermotolerance of PpHSF5-OE lines.

\section{Discussion}

\section{Peach Contains Fewer HSF Gene Family Members among Several Plant Species}

HSFs play vital roles in plant growth and defense. Through plant genome sequencing, $H S F$ gene family members have been identified in several model organisms and more than 20 plant species (Supplemental Table S8). Only a single $H S F$ was detected in yeast, nematodes and flies (Nakai,1999; Nover,1996). In this study, 18 HSF genes were identified in peach, which is less than in most other plant species, but more than in tea (Camellia sinensis), strawberry (Fragaria vesca), Chinese plum (Prunus salicina) and carnation (Dianthus caryophyllus) (Hu et al., 2015;

Liu et al., 2016; Qiao et al., 2015; Li et al., 2019). 
341 C. The same phenomenon was also observed in peach, which contained 11 HSFAs, six HSFBs 342 and one HSFCs.

343 The HSF Gene Family Expanded along with DSD in Peach

344 The number of HSFs expanded markedly during plant evolution. The analysis of 51

345 representative species indicated that the HSF gene family largely expanded along with WGD 346 during plant evolution (Wang et al., 2018). In Chinese white pear (Pyrus bretschneideri), most 347 PbHSF expansions dated back to a recent WGD (Qiao et al., 2015). On the other hand, GmHSFS 348 in cotton expanded along single gene duplication events (Wang et al., 2014). Here, DSD (67\%) was the primary type of duplication for the HSF gene family in peach. The same phenomenon was also seen for the E3 ligase gene family in peach (Tan et al., 2019). It is probably that peach has not undergone a recent WGD (Verde et al., 2013).

HSF Gene Family was Classified into Three Classes

Plant HSF proteins contain a few conserved characteristic domain (Guo et al., 2016). Generally, HSF families in plant species can be divided into three subfamilies, termed HSFA, HSFB, and HSFC (Liu et al., 2018; Wang et al., 2018). The classification of the PpHSF family was consistent with that in other plant species (Supplemented Table 7). Multiple sequence alignments revealed that an insertion occurred in the DBD domain near the N-terminus in the PpHSFA and PpHSFC groups. Like in other plants, the PpHSFA and PpHSFC genes contained inserted coding sequence for 21 and 7 aa in the HR-A/B region, respectively, while the HR-A/B region of 
360

361

362

363

364

PpHSFB was compact (Nover, 1996; Scharf et al., 2012). The organization, composition, number of conserved motifs in the HSFs differed among plant species (Wang et al., 2018). In Chinese whit pear, Class A in PbHSFs contained the most conversed motifs, followed by class B and then class C (Qiao et al., 2015). In this study, the number of motifs in the different classes was consistent with those in Chinese white pear. This also showed that members of the same class often have similar sequence structures in peach. For example, motif 5 was present only in PpHSFA and PpHSFC, while all Class B and Class A HSFs contain motif 4. The presence of these motifs may lead to functional group specificity. The similar classifications of HSF families in diverse plants showed that the HSF family was highly conserved during long-term evolution.

\section{PpHSF5 Acts as Repressor of Organ Size in Plants}

In plants, organ size is primarily controlled by internal developmental signals (Mizukami, 2001;

Dubrovsky et al., 2006; Spradling , 2001; West et al., 2004). Previous research in the model organism Arabidopsis thaliana indicates that plant hormones and transcription factors, including HSFs, play crucial roles in growth and development (Petricka et al., 2012; Begum et al., 2013). HSFs as key transcription factors protect plants from various abiotic stresses and then participate in the growth and development (Guo et al., 2016). For example, OsHsfAla, OsHsfA1b and OsHsfAld are the main positive regulators of gene expression on heat stress-responsive, and four HSFA proteins play significant roles in gene expression of plant growth and development (Yoshida et al., 2011). In poplar (Populus trichocarpa), the transcripts of three PtHsfs in the B4 subfamily (-B4b, -B4c and -B4d) were maintained at higher levels during the leaf expansion 
380

381

382

383

384

385

386

387

388

389

390

391

392

393

394

395

396

399

397 It is still unknown how PpHSF5 regulates root and aerial organs development. PpHSF5 is

398 homologous to AtHSFB4 and thus may play similar roles in root development. Confocal laser

stages (Liu et al., 2019). In carnation (Dianthus caryophyllus), five DcaHsfs, namely DcaHsf-A1,

$A 2 a, A 9 a, B 2 a, B 3 a$, were involved in early flowering stages (Li et al., 2019). Transgenic

Arabidopsis plants overexpressing AtHSFB4 contained massively enhanced levels of AtHSFB4

mRNAs and exhibited shorter roots (Begum et al., 2013). In this study, overexpression of

35S:PpHSF5 in Arabidopsis resulted in not only shorter roots but also in lesser root volume and

fewer lateral roots and root forks compared to WT.

The root system of a plant is instrumental to its growth and productivity because it is

responsible for the extraction of water and mineral nutrients from the soil and their transport to

aboveground parts of the plant (Hochholdinger, 1998). In this study, the 35S:PpHSF5 transgenic

lines produced smaller aerial organs compared with WT. For example, the size (length and width)

of rosette leaves were smaller than WT two and three weeks after transplanting, while the

number of rosette leaves was not affected. The height of the overexpression lines was

significantly lower than that in WT, while the number of internodes was not. Overexpression of

OsHsfA2e in rice caused a dwarf phenotype (Yokotani et al., 2008). In plants overexpressing

$B h H s f 1$, the reduced organ size was mainly attributed to decreased cell proliferation (Zhu et al.,

2009). The overexpression of PpHSF5 in peach suggested that the dwarf phenotype of transgenic

plants was caused by shorter internodes. scanning of roots in AtHSFB4-overexpression transgenic lines showed that ectopic division of 
400 the lateral root cap cells (LRC) occurred (Begum et al., 2013). Previous studies indicated that

401 auxin acts in the production of lateral root primordium (LR) (Casimiro et al., 2003; West et al.,

402 2004). In the promoter of PpHSF5, there are three cis-acting regulatory elements that contain the

403 auxin-inducible TGACG-motif. Two auxin-inducible TGA-box elements in the GmGH3

404 promoter were strong binding sites of plant nuclear proteins and improved the auxin inducibility

405 of the GmGH3 promoter (Zhan-Bin et al., 1994). Moreover, the HS assays indicated PpHSF5-

406 OE lines exhibited enhanced thermotolerance compared to WT. Similarity results were observed

407 in transgenic Arabidopsis plants with AtHsfA3 and rice plants with OsHsfA2e (Ogawa et al.,

408 2007; Yokotani et al., 2008). Therefore, PpHSF5 might be as a responsive factor for temperature

409 change and involved in auxin signal transduction due to the TGA motifs in its promoter and

410 might serve to negatively regulate root elongation and lateral root development, ultimately

411 affecting the growth of aboveground parts of the plant.

413 Conclusions

414 In this report, $18 \mathrm{PpHSF}$ genes were discovered in peach and found to be nonuniformly

415 distributed on the peach chromosomes. The PpHSF family could be classified into three classes

416 (PpHSFA, PpHSFB and PpHSFC) through multiple alignment, motif analysis and phylogenetic

417 comparison. The expansion of the HSF gene family in peach occurred through DSD (67\%) and

418 WGD (33\%). PpHSF5 was expressed in diverse tissues and organs of the peach cultivar

419 'Zhongyoutao 14', with higher levels in young vegetative organs (leaf and apex). Transgenic

Peer] reviewing PDF | (2020:02:45572:5:0:NEW 10 Jan 2021) 
420 Arabidopsis lines overexpressing PpHSF5 showed massively enhanced levels of PpHSF5.

421 Ectopic expression PpHSF5 repressed the length and number of roots, length and width of

422 rosette leaves, and the height of plants, and enhanced thermtolerance in Arabidopsis after heat

423 stress treatment. Our results further supplied functional and annotation information of the HSF

424 gene family in general and revealed potential roles, outside of the response to heat stress, for

425 PpHSF5 during plant development.

\section{Acknowledgements}

The authors would like to thank Anita K. Snyder for critical reading the manuscript.

References

Begum T, Reuter R, Schoffl F. 2013. Overexpression of AtHsfB4 induces specific effects on root development of Arabidopsis. Mech Dev, 130(1): 54-60 DOI: 10.1016/j.mod.2012.05.008.

Busch W, Wunderlich M, Schoffl F. 2005. Identification of novel heat shock factor-dependent genes and biochemical pathways in Arabidopsis thaliana. Plant Journal, 41(1):1-14

DOI: 10.1111/j.1365-313X.2004.02272.x.

Casimiro I, Beeckman T, Graham N, Bhalerao R, Zhang H, Casero P, Bennett MJ. 2003. Dissecting Arabidopsis lateral root development. Trends in Plant Science, 8(4): 165-171.

DOI: $10.1016 / \mathrm{s} 1360-1385(03) 00051-7$.

Chan-Schaminet KY, Baniwal SK, Bublak D, Nover L, Scharf KD. 2009. Specific interaction between tomato HsfA1 and HsfA2 creates hetero-oligomeric superactivator complexes for synergistic activation of heat stress gene expression. Journal of Biological Chemistry, 284(31): 20848-20857 
DOI: 10.1074/jbc.M109.007336.

Chauhan H, Khurana N, Agarwal P, Khurana P. 2011. Heat shock factors in rice (Oryza sativa L.): Genomewide expression analysis during reproductive development and abiotic stress. Molecular Genetics and Genomics, 286(2): 171-187 DOI: 10.1007/s00438-011-0638-8.

Chen C, Chen H, Zhang Y, Thomas H R, Frank M H, He Y, Xia R. 2020. TBtools: An Integrative Toolkit Developed for Interactive Analyses of Big Biological Data[J]. Molecular Plant, 13(8):1194-1202. DOI: $10.1016 /$ j.molp.2020.06.009

Chung MH, Chen MK, Pan SM. 2000. Floral spray transformation can efficiently generate Arabidopsis transgenic plants. Transgenic Research, 9(6):471-486 DOI: 10.1023/A:1026522104478.

Dubrovsky JG, Gambetta GA, Hernandez-Barrera A, Shishkova S, Gonzalez I. 2006. Lateral root initiation in Arabidopsis: developmental window, spatial patterning, density and predictability. Annals of Botany, 97(5): 903-915 DOI: 10.1093/aob/mcj604.

Finn RD, Coggill P, Eberhardt RY, Eddy SR, Mistry J, Mitchell AL, Potter SC, Punta M, Qureshi M, Sangrador-Vegas A, Salazar GA, Tate J, Bateman A. The Pfam protein families database: towards a more sustainable future. Nucleic acids research, 44(D1): 279-285 DOI: 10.1093/nar/gkv1344.

Guo M, Liu JH, Ma X, Luo DX, Gong ZH, Lu MH. 2016. The plant heat stress transcription factors (HSFs): structure, regulation, and function in response to abiotic stresses. Frontiers in Plant Science, 7: 114-127 DOI: $10.3389 /$ fpls.2016.00114.

Guo M, Lu JP, Zhai YF, Chai WG, Gong ZH, Lu MH. 2015. Genome-wide analysis, expression profile of heat shock factor gene family (CaHsfs) and characterisation of CaHsfA2 in pepper (Capsicum annuum L.). BMC Plant Biology, 15(1): 151-171 DOI: 10.1186/s12870-015-0512-7.

Hahn A, Bublak D, Schleiff E, Scharf KD. 2011. Crosstalk between Hsp90 and Hsp70 chaperones and heat stress transcription factors in tomato. Plant Cell, 23(2): 741-755 DOI: 10.1105/tpc.110.076018.

He Y, Li W, Lv J, Jia Y, Wang M, Xia G. 2012. Ectopic expression of a wheat MYB transcription factor gene, TaMYB73, improves salinity stress tolerance in Arabidopsis thaliana. Journal of Experimental Botany, 63(3): 1511-1522 DOI: $10.1093 / \mathrm{jxb} / \mathrm{err} 389$.

Hochholdinger F, Gunter Feix. 1998. Early post-embryonic development is specifically affected in the maize mutant lrt1. The Plant Journal, 16(2): 247-255 DOI: 10.1046/j.1365-313x.1998.00280.x.

Hu W, Hu G, Han B. 2009. Genome-wide survey and expression profiling of heat shock proteins and heat shock 
471

472

473

474

475

476

477

478

479

480

481

482

483

484

485

486

487

488

489

490

491

492

493

494

495

496

497

498

499

500

factors revealed overlapped and stress specific response under abiotic stresses in rice. Plant Science, 176(4): 583-590 DOI: 10.1016/j.plantsci.2009.01.016.

Hu Y, Han YT, Wei W, Li YJ, Zhang K, Gao YR, Feng JY. 2015. Identification, isolation, and expression analysis of heat shock transcription factors in the diploid woodland strawberry Fragaria vesca. Frontiers in Plant Science, 6: 736-751 DOI: 10.3389/fpls.2015.00736.

Kotak S, Vierling E, Baumlein H, von Koskull-Doring P. 2007. A novel transcriptional cascade regulating expression of heat stress proteins during seed development of Arabidopsis. Plant Cell, 19(1): 182-195 DOI: 10.1105/tpc.106.048165.

Koskull-Doring vP, Scharf KD, Nover L. 2007. The diversity of plant heat stress transcription factors. Trends Plant Science, 12(10): 452-457 DOI: 10.1016/j.tplants.2007.08.014.

Krzywinski M, Schein J, Birol I, Connors J, Gascoyne R, Horsman D, Marra, MA. 2009. Circos: An information aesthetic for comparative genomics. Genome Research, 19(9): 1639-1645 DOI: 10.1101/gr.092759.109.

Kumar M, Busch W, Birke H, Kemmerling B, Nurnberger T, Schoffl F. 2009. Heat shock factors HsfB1 and HsfB2b are involved in the regulation of Pdf1.2 expression and pathogen resistance in Arabidopsis. Molecular Plant, 2(1): 152-165 DOI: 10.1093/mp/ssn095.

Li PS, Yu TF, He GH, Chen M, Zhou YB, Chai SC, Ma YZ. 2014. Genome-wide analysis of the Hsf family in soybean and functional identification of GmHsf-34 involvement in drought and heat stresses. BMC Genomics, 15(1): 1009-1025 DOI: 10.1186/1471-2164-15-1009.

Li W, Cui X, Meng Z, Huang X, Xie Q, Wu H, Liang W. 2012. Transcriptional regulation of Arabidopsis MIR168a and ARGONAUTE1 homeostasis in abscisic acid and abiotic stress responses. Plant Physiol, 158(3): 1279-1292 DOI: 10.1104/pp.111.188789.

Li W, Wan X L, Yu J Y, Wang K L, Zhang J. 2019. Genome-wide identification, classification, and expression analysis of the Hsf gene family in Carnation (Dianthus caryophyllus). International Journal of Molecular Sciences, 20(20): 5233-5251 DOI: 10.3390/ijms20205233.

Lian X D, Tan B, Yan L, Jiang C, Cheng J, Zheng X B, Feng J C. 2020. Transcript profiling provides insights into molecular processes during shoot elongation in temperature-sensitive peach (Prunus persica). Scientific Reports, 10(1): 7801-7813 DOI: 10.1038/s41598-020-63952-2.

Liu G, Chai F, Wang Y, Jiang J, Duan W, Wang Y, Wang L. 2018. Genome-wide identification and classification of HSF family in Grape, and their transcriptional analysis under heat acclimation and heat 
501

502

503

504

505

506

507

508

509

510

511

512

513

514

515

516

stress. Horticultural Plant Journal, 4(4): 133-143 DOI: 10.1016/j.hpj.2018.06.001.

Liu M, Huang Q, Sun W, Ma Z, Huang L, Wu Q, Tang, Z, Bu T, Li C, Chen H. 2019. Genome-wide investigation of the heat shock transcription factor (Hsf) gene family in Tartary buckwheat (Fagopyrum tataricum). BMC Genomics, 20(1): 871-888 DOI: 10.1186/s12864-019-6205-0.

Liu ZW, Wu ZJ, Li XH, Huang Y, Li H, Wang YX, Zhuang J. 2016. Identification, classification, and expression profiles of heat shock transcription factors in tea plant (Camellia sinensis) under temperature stress. Gene, 576(1 Pt 1): 52-59 DOI: 10.1016/j.gene.2015.09.076.

Liu B, Hu J, Zhang J. 2019. Evolutionary divergence of duplicated Hsf genes in Populus. Cells, 8(5): 438-463 DOI: 10.3390/cells8050438.

Lu ZH, Niu L, Chagné D, Cui GC, Pan L, Foster T, Zhang RP, Zeng WF, Wang ZQ. 2016. Fine mapping of the temperature-sensitive semi-dwarf (Tssd) locus regulating the internode length in peach (Prunus persica). Molecular Breeding, 36(2). DOI: 10.1007/s11032-016-0442-6

Nakai A. 1999. New aspects in the vertebrate heat shock factor system: Hsf3 and Hsf4. Cell Stress and Chaperones, 4(2): 86-93 DOI: $10.1054 / \mathrm{csac} .1998 .0002$.

Nishizawa-Yokoi A, Nosaka R, Hayashi H, Tainaka H, Maruta T, Tamoi M, Shigeoka S. 2011. HsfA1d and HsfAle involved in the transcriptional regulation of $H_{s f A 2}$ function as key regulators for the Hsf signaling network in response to environmental stress. Plant Cell Physiol, 52(5): 933-945 DOI: 10.1093/pcp/pcr045.

Nishizawa A, Yabuta Y, Yoshida E, Maruta T, Yoshimura K, Shigeoka S. 2006. Arabidopsis heat shock transcription factor A2 as a key regulator in response to several types of environmental stress. The Plant Journal, 48(4): 535-547 DOI: 10.1111/j.1365-313X.2006.02889.x.

Nover L. 1996. The Hsf world: classification and properties of plant heat stress transcription factors. Cell Stress and Chaperones, 1(4): 215-224.DOI: 10.1379/1466-1268(1996)001<0215:THWCAP>2.3.CO;2.

Nover L, Bharti K, Doring P, Mishra SK, Ganguli A, Scharf KD. 2001. Arabidopsis and the heat stress transcription factor world: how many heat stress transcription factors do we need? Cell Stress Chaperones, 6(3): 177-189 DOI: $10.2307 / 1601759$.

Ogawa D, Yamaguchi K, Nishiuchi T. 2007. High-level overexpression of the Arabidopsis HsfA2 gene confers not only increased themotolerance but also salt/osmotic stress tolerance and enhanced callus growth. J Exp Bot, 58(12), 3373-3383. DOI: 10.1093/jxb/erm184

Petricka JJ, Winter CM, Benfey PN. 2012. Control of Arabidopsis root development. Annual Review of Plant Biology, 63(1): 563-590 DOI: 10.1146/annurev-arplant-042811-105501. 
531

532

533

534

535

536

537

538

539

540

541

542

543

544

545

546

547

548

549

550

551

552

553

554

555

556

557

558

559

560

Qiao X, Li M, Li L, Yin H, Wu J, Zhang S. 2015. Genome-wide identification and comparative analysis of the heat shock transcription factor family in Chinese white pear (Pyrus bretschneideri) and five other Rosaceae species. BMC Plant Biology, 15(1): 12-28 DOI: 10.1186/s12870-014-0401-5.

Scharf KD, Berberich T, Ebersberger I, Nover L. 2012. The plant heat stress transcription factor (Hsf) family: structure, function and evolution. Biochim et Biophys Acta, 1819(2): 104-119

DOI: 10.1016/j.bbagrm.2011.10.002.

Schmidt R, Schippers JHM, Welker A, Mieulet D, Guiderdoni E, Mueller-Roeber B. 2012. Transcription factor OsHsfC1b regulates salt tolerance and development in Oryza sativa ssp. japonica. AoB PLANTS, 2012: 117 DOI: $10.1093 /$ aobpla/pls011.

Singh A, Mittal D, Lavania D, Agarwal M, Mishra RC, Grover A. 2012. OsHsfA2c and OsHsfB4b are involved in the transcriptional regulation of cytoplasmic OsClpB (Hsp100) gene in rice (Oryza sativa L.). Cell Stress Chaperones, 17(2): 243-254 DOI: 10.1007/s12192-011-0303-5.

Schmittgen T D, Livak K J. 2008. Analyzing real-time PCR data by the comparative $\mathrm{C}(\mathrm{T})$ method. Nature Protocols, 3(6): 1101-1108 DOI: 10.1038/nprot.2008.73.

Sparkes IA, Runions J, Kearns A, Hawes C. 2006. Rapid, transient expression of fluorescent fusion proteins in tobacco plants and generation of stably transformed plants. Nature Protocols, 1(4): 2019-2025 DOI: 10.1038/nprot.2006.286.

Spradling A, Drummond-Barbosa D, Kai T. 2001. Stem cells find their niche. Nature, 414(6859): 98-104 DOI: $10.1038 / 35102160$.

Tan B, Lian X, Cheng J, Zeng W, Zheng X, Wang W, Feng J. 2019. Genome-wide identification and transcriptome profiling reveal that E3 ubiquitin ligase genes relevant to ethylene, auxin and abscisic acid are differentially expressed in the fruits of melting flesh and stony hard peach varieties. BMC Genomics, 20(1): 892-906 DOI: 10.1186/s12864-019-6258-0.

Verde I, Abbott AG, Scalabrin S, Jung S, Shu S, Rokhsar DS. 2013. The high-quality draft genome of peach (Prunus persica) identifies unique patterns of genetic diversity, domestication and genome evolution. Nature Genetics, 45(5): 487-494 DOI: 10.1038/ng.2586.

Wang J, Sun N, Deng T, Zhang L, Zuo K. 2014. Genome-wide cloning, identification, classification and functional analysis of cotton heat shock transcription factors in cotton (Gossypium hirsutum). BMC Genomics, 15(1): 961-979 DOI: 10.1186/1471-2164-15-961.

Wang X, Shi X, Chen S, Ma C, Xu S. 2018. Evolutionary origin, gradual accumulation and functional divergence 
561

562

563

564

565

566

567

568

569

570

571

572

573

574

575

576

577

578

579

580

581

582

583

584

585

586

587

588

589

of heat shock factor gene family with plant evolution. Frontiers in Plant Science, 9: 71-87. DOI: 10.3389/fpls.2018.00071.

Wang Y, Tang H, Debarry JD, Tan X, Li JP, Wang XY, Lee T, Jin HZ, Marler B, Guo H, Kissinger JC, Paterson AH. 2012. MCScanX: a toolkit for detection and evolutionary analysis of gene synteny and collinearity. Nucleic Acids Res, 40(7), e49. DOI: 10.1093/nar/gkr1293

West G, Inzé D, Beemster GTS. 2004. Cell cycle modulation in the response of the primary root of Arabidopsis to salt stress. Plant Physiol, 135(2): 1050-1058 DOI: 10.1104/pp.104.040022.

Xue GP, Sadat S, Drenth J, McIntyre CL. 2014. The heat shock factor family from Triticum aestivum in response to heat and other major abiotic stresses and their role in regulation of heat shock protein genes. Journal of Experimental Botany, 65(2): 539-557 DOI: 10.1093/jxb/ert399.

Yang Z, Wang Y, Gao Y, Zhou Y, Zhang E, Hu Y, Xu C. 2014. Adaptive evolution and divergent expression of heat stress transcription factors in grasses. BMC Evolutionary Biology, 14(1), 147-158 DOI: $10.1186 / 1471-2148-14-147$.

Yokotani N, Ichikawa T, Kondou Y, Matsui M, Hirochika H, Iwabuchi M, Oda K. 2007. Expression of rice heat stress transcription factor OsHsfA2e enhances tolerance to environmental stresses in transgenic Arabidopsis. Planta, 227(5), 957-967. DOI: 10.1007/s00425-007-0670-4

Yoshida T, Ohama N, Nakajima J, Kidokoro S, Mizoi J, Nakashima K, Yamaguchi-Shinozaki K. 2011. Arabidopsis $H_{s f A l}$ transcription factors function as the main positive regulators in heat shock-responsive gene expression. Molecular Genetics and Genomics, 286(5-6): 321-332

DOI: $10.1007 / \mathrm{s} 00438-011-0647-7$.

Yu X Y, Yao Y, Hong Y H, Hou P Y, Li C X, Xia Z Q, Chen Y H. 2019. Differential expression of the Hsf family in cassava under biotic and abiotic stresses. Genome, 62(8): 563-569 DOI: 10.1139/gen-2018-0163.

Zhan-Bin Liu TU, Xiangyang Shi, Gretchen Hagen, and Tom J. Guilfoyle. 1994. Soybean $G H 3$ promoter contains multiple auxin-inducible elements. The Plant Cell, 6(5): 645-657 DOI: 10.1105/tpc.6.5.645.

Zhang J, Jia H, Li J, Li Y, Lu M, Hu J. 2016. Molecular evolution and expression divergence of the Populus euphratica Hsf genes provide insight into the stress acclimation of desert poplar. Scientific Reports, 6(1): 30050-30063 DOI: 10.1038/srep30050.

Zhu X, Huang C, Zhang L, Liu H, Yu J, Hu Z, Hua W. 2017. Systematic analysis of Hsf family genes in the Brassica napus genome reveals novel responses to heat, drought and high $\mathrm{CO}_{2}$ stresses. Frontiers in Plant 
Figure 1

Chromosomal location of HSF genes in peach (PpHSFs).

Three syntenic pairs are linked by red lines. 


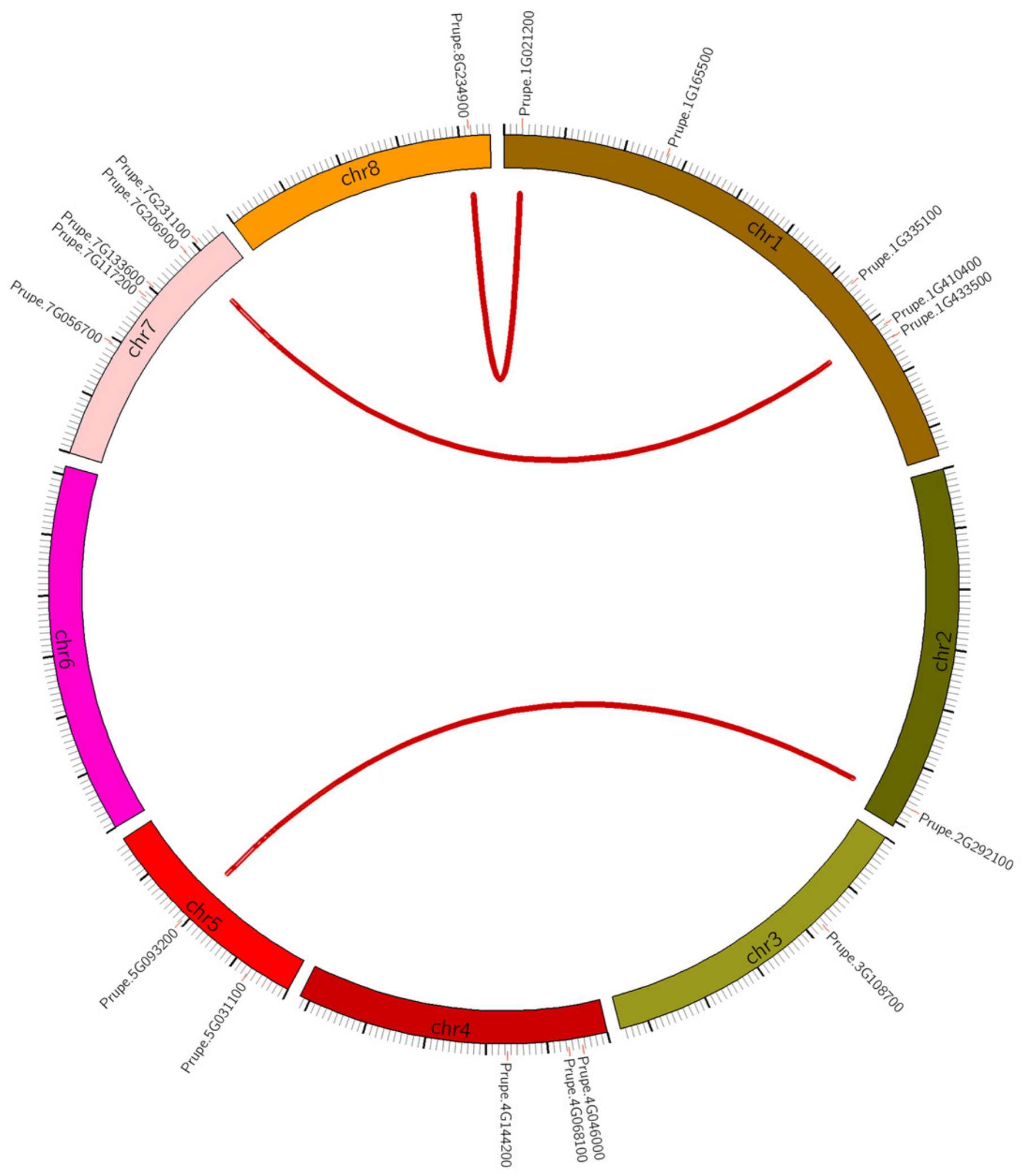




\section{Figure 2}

Multiple sequence alignment of the HR-A/B regions (OD), conserved motif and phylogenetic analysis of PpHSFs

A. Multiple sequence alignment of the HR-A/B regions, from the start of the DNA-binding domain to the end of the HR-A/B region, of the HSF proteins were aligned with MEGA 6. B. hylogenetic tree of HSFs from Prunus persica (Pp, red star), Oryza sativa (Os, blue circle) and Arabidopsis thaliana (At, green square) constructed by maximum likelihood method (ML) with Jones-Taylor-Thornton (JTT) model in MEGA 6.0. Both locus ID and subclass numbers are listed. C. Analysis of conserved motifs in the HSF gene family in peach. Proteins are organized according to the groups in Figure 2A. Ten motifs were found in the protein sequences as shown in Supplemental Table S4. 


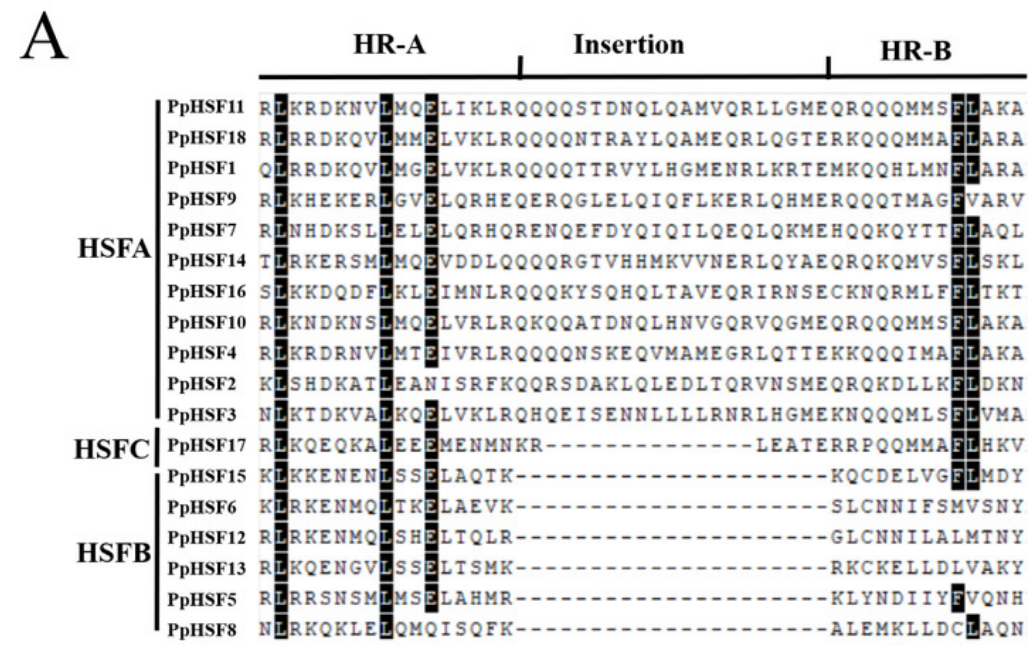

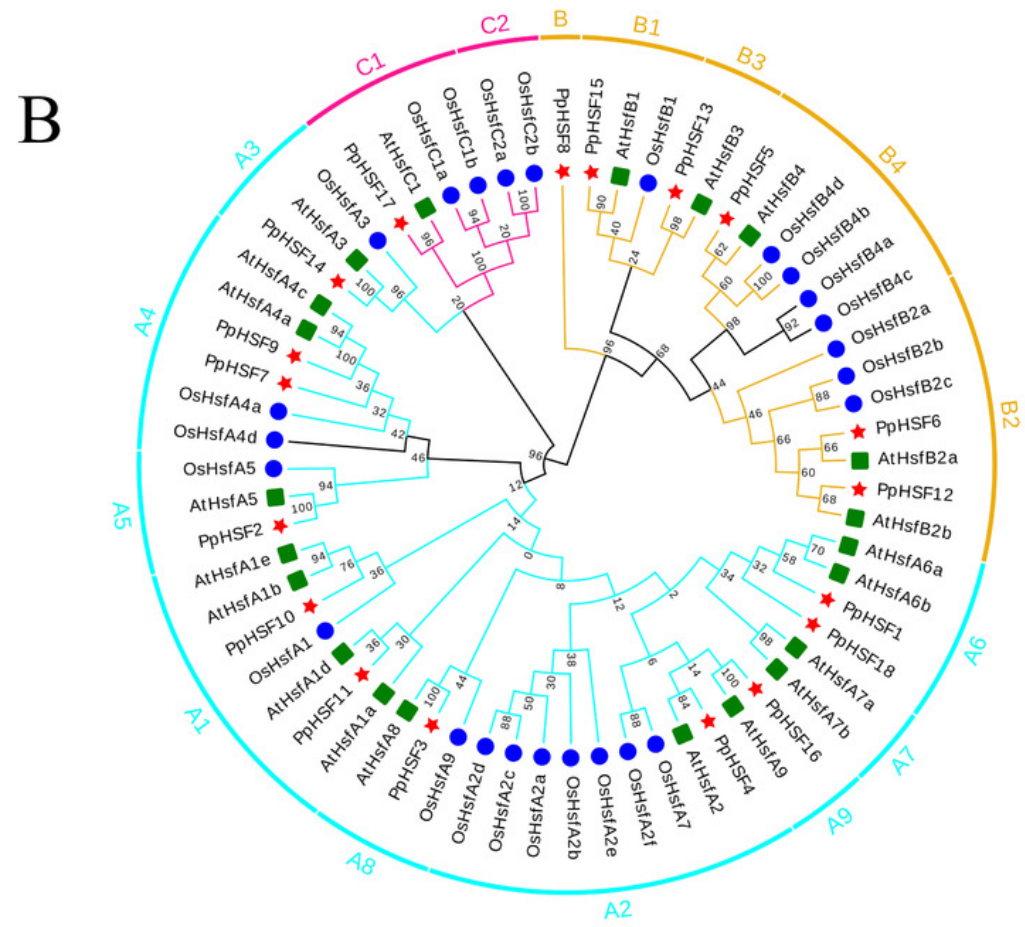

C

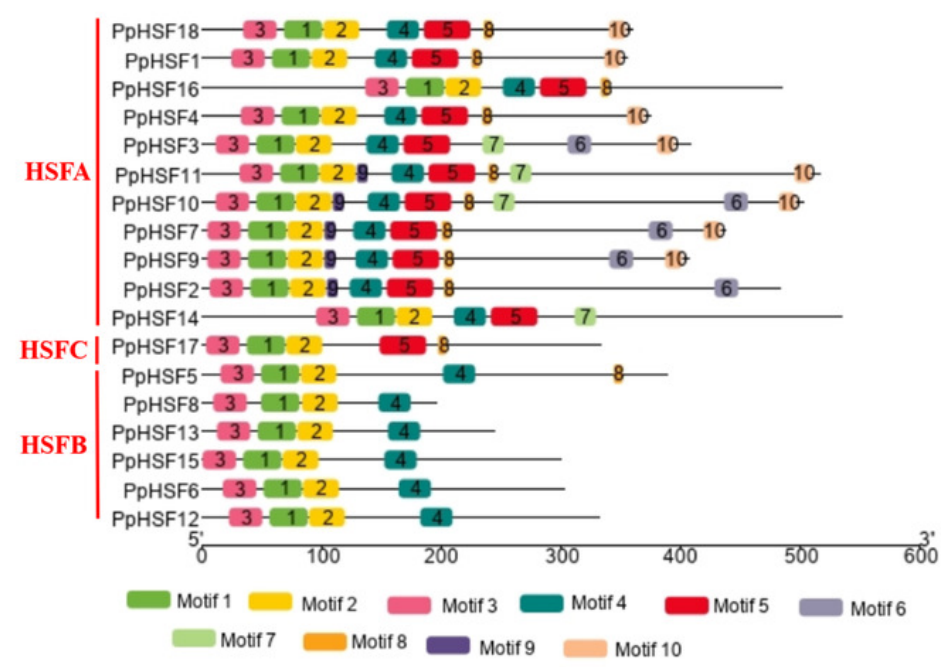

Peer) reviewing PDF | (2020:02:45572:5:0:NEW 10 Jan 2021) 


\section{Figure 3}

Heatmap of transcript levels of HSF genes in peach.

Transcriptome data were used to measure the expression level of PpHSFs. The gene names on the right are organized according to the different subclasses. Samples were harvest from shoots at the IP (initial period), IEP (initial elongation period), RGP (rapid growth period), and SGP (stable growth period), which are four key growth stages during temperature-sensitive peach shoot development. Color scale at the top represents FPKM values. Blue indicates low expression and red indicates high expression. Heatmap was generated using TBtools. 


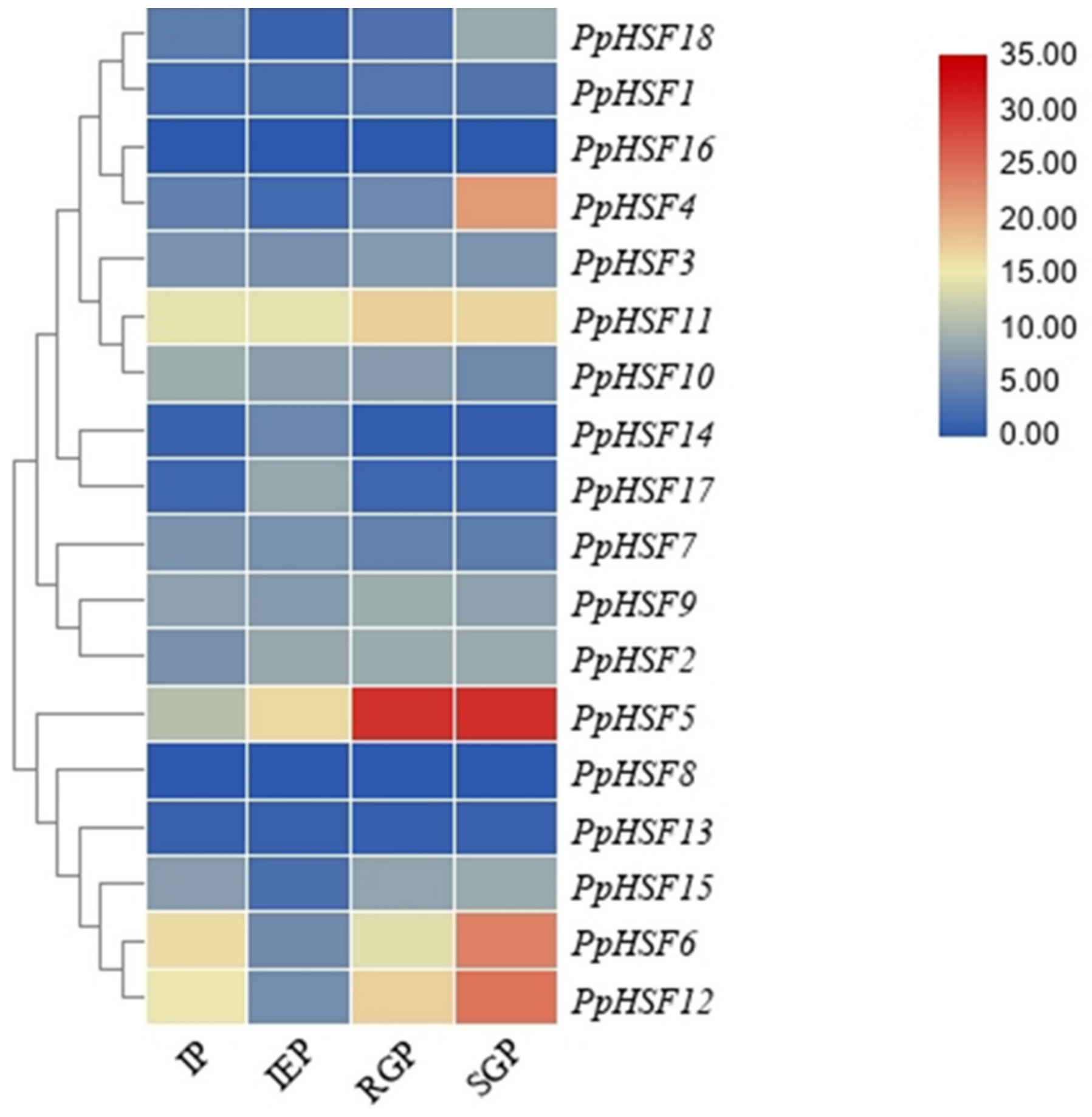


Figure 4

Relative expression of PpHSF5 in different tissues of 'Zhongyoutao 14' peach.

Established plants were grown under normal conditions. The analyzed tissues include the apex, flower, embryo, young leaf, and mature leaf, which harvested at the same time. The relative expression levels were calculated using the $2^{-\Delta \Delta C T}$ method.

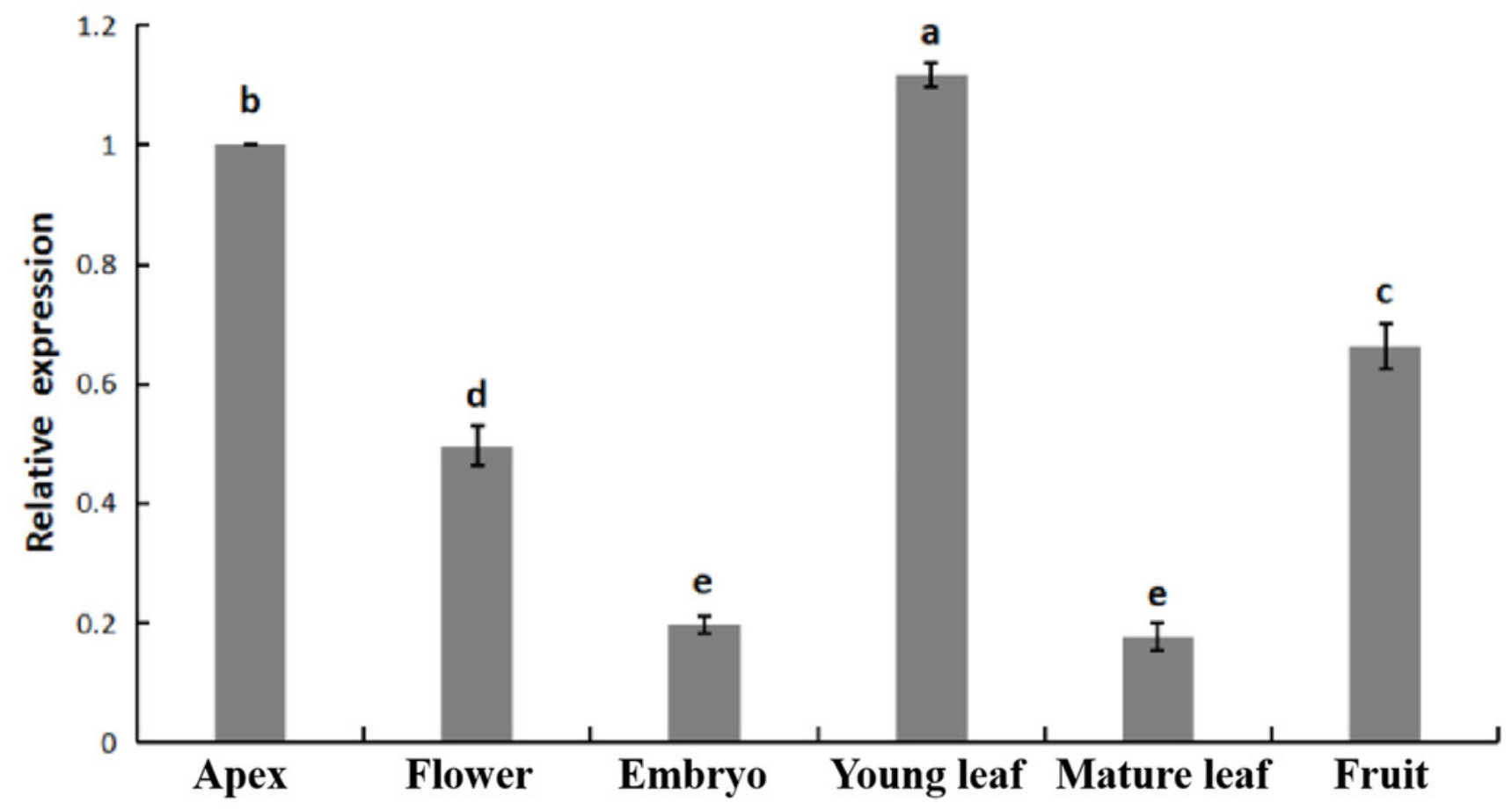




\section{Figure 5}

Subcellular localization of PpHSF5 in $N$. benthamiana epidermal cells.

A and $\mathbf{D}$ : Images of green fluorescence from the GFP protein and the PpHSF5-GFP fusion protein in tobacco cells under the confocal microscope; B and E: Bright field image of tobacco epidermal cells; C: Overlay of A and B; F: Overlay of D and E.

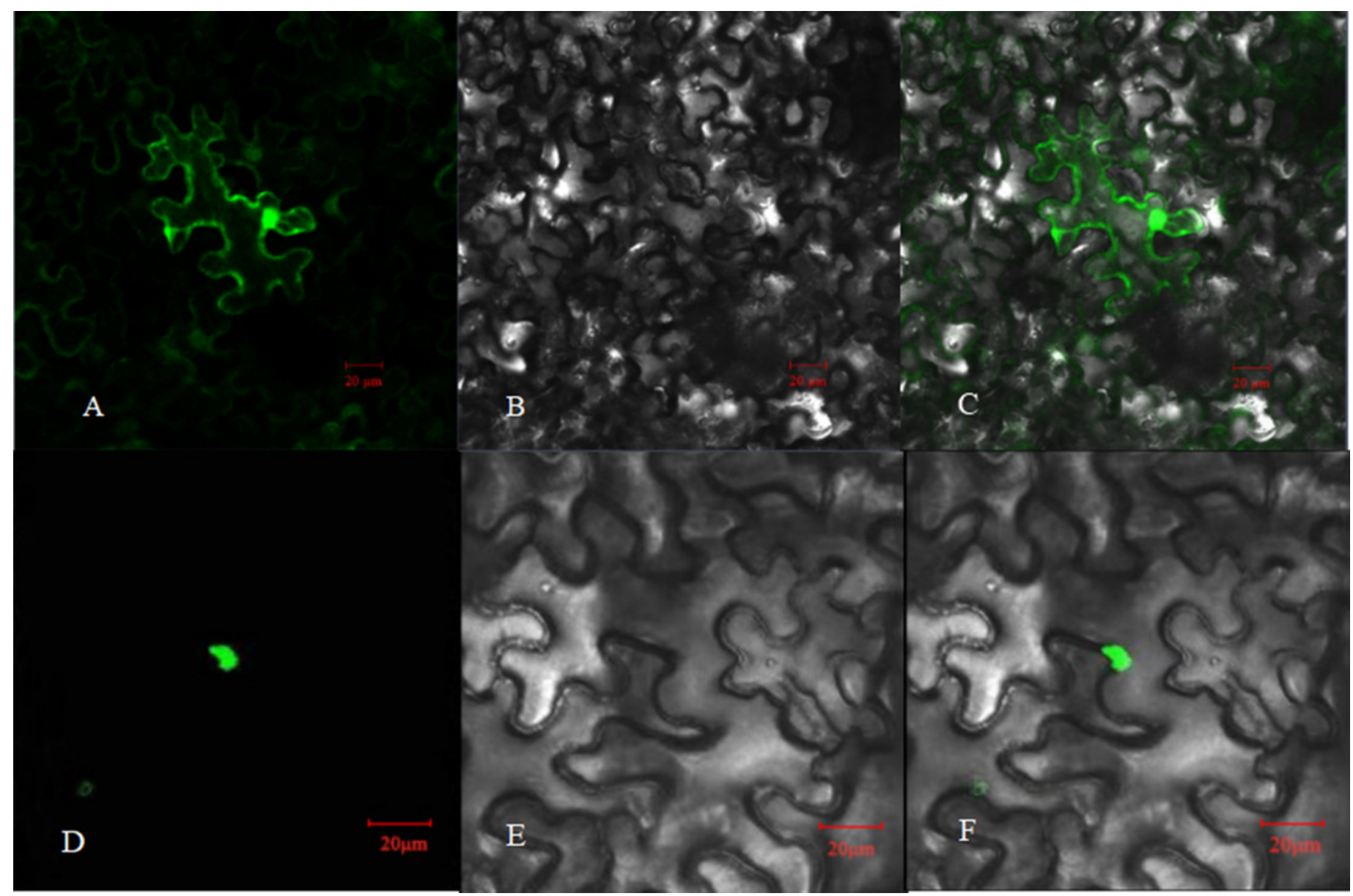




\section{Figure 6}

Phenotypic and expression analysis of transgenic Arabidopsis over-expressing PpHSF5

A. Phenotype of $\mathrm{T}_{2}$ transgenic plants from two lines over-expressing PpHSF5 after cultivation for one week. B. Root length of $\mathrm{T}_{2}$ transgenic plants over-expressing PpHSF5. Three plants were measured in each biological replicate. $\mathbf{C}$. Phenotype of $\mathrm{T}_{2}$ transgenic plants from two lines over-expressing PpHSF5 after cultivation for two weeks. Seeds were transferred to soil after germination and growth on agar for five days. $\mathbf{D}$. The morphology and relative expression of $\mathrm{T}_{2}$ transgenic plants with PpHSF5 and WT after cultivation for two weeks. The number of rosettes (a), length (b) and width (c) after cultivation for two weeks. Relative expression of PpHSF5 in transgenic Arabidopsis plants carrying p35S:PpHSF5 (d); E. Phenotype of $\mathrm{T}_{2}$ transgenic plants over-expressing PpHSF5 after cultivation in soil for three weeks. $\mathbf{F}$. The length, width and number of rosette leaves, number of internodes and flower stalks, and the height of plants after cultivation for three weeks. G. Phenotype of $T_{2}$ transgenic plant roots over-expressing PpHSF5 after cultivation for three weeks. $\mathbf{H}$. The root length, volume and other indexes were scanned after cultivation for three weeks. 
A

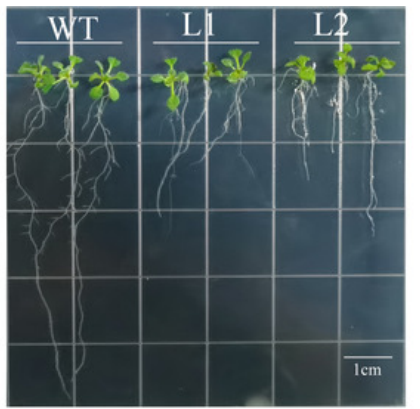

C

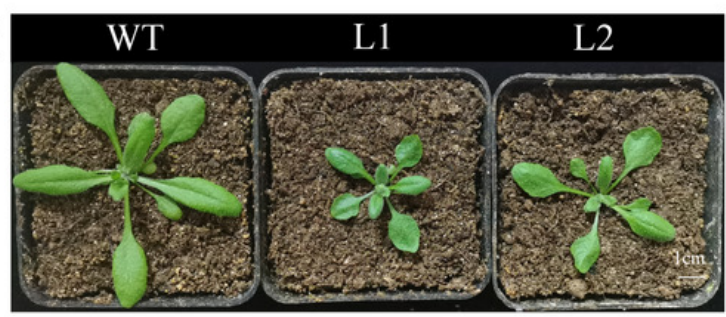

B

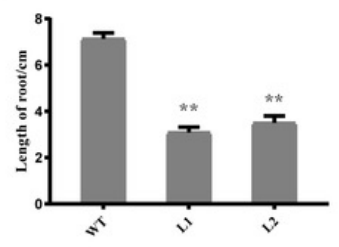

D
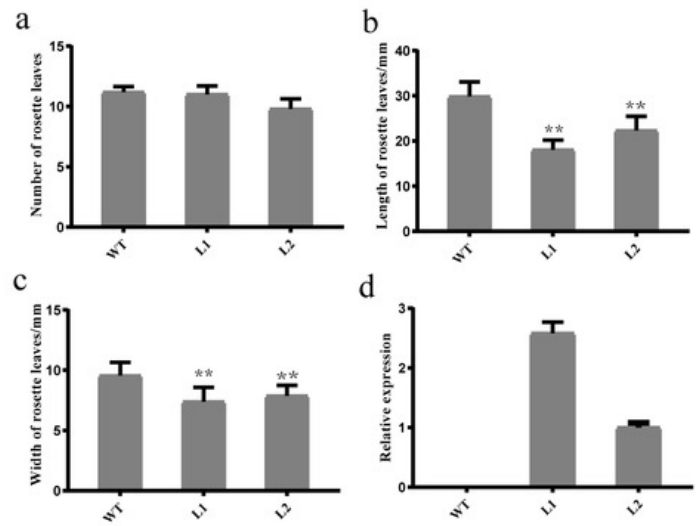

E
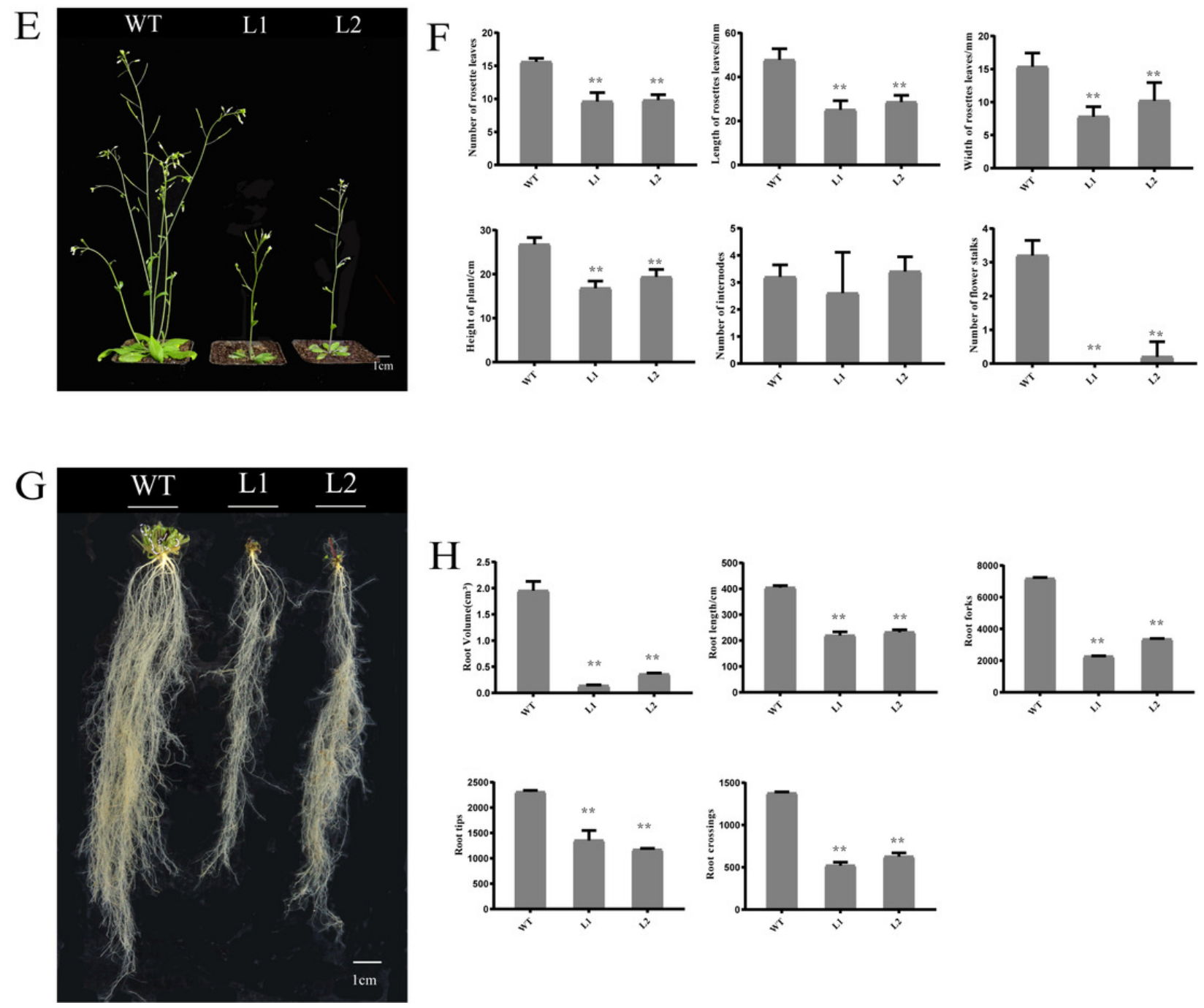

Peer) reviewing PDF | (2020:02:45572:5:0:NEW 10 Jan 2021) 


\section{Figure 7}

Thermotolerance of the p35S::PpHSF5 plants.

A: Five-day-old seedlings of wild type and the p35S::PpHSF5 plants were treated at $46{ }^{\circ} \mathrm{C}$ for 30 min. Photographs were taken before HS treatment. B. Photographs were taken after 1d in $22{ }^{\circ} \mathrm{C}$. C: Photographs were taken after $3 \mathrm{~d}$ in $22^{\circ} \mathrm{C}$. D: Photographs were taken after $5 \mathrm{~d}$ in $22{ }^{\circ} \mathrm{C}$.E: Photographs were taken after $7 \mathrm{~d}$ in $22^{\circ} \mathrm{C}$. F: Comparison of germination rate among wild-type, p35S::PpHSF5 transgenetic plants after HS treatment. The number of germinated plants was counted daily after HS treatment. For three replication, more than 50 seedlings were used each lines ( $t$-test signifificant at $P<0.05$ and $P<0.01$, respectively).

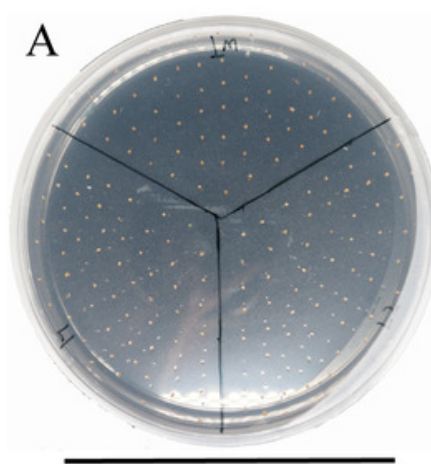

before HS

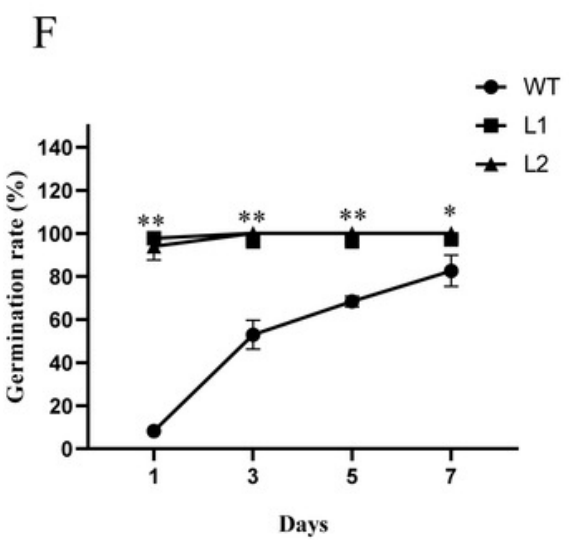

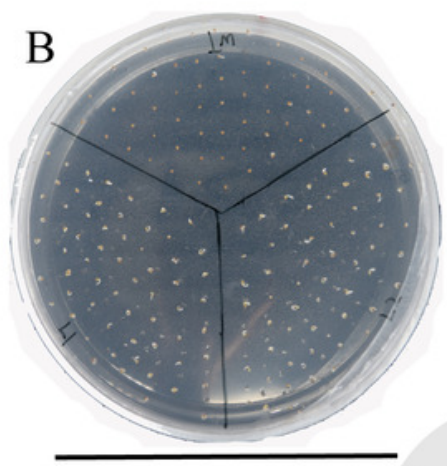

$1 \mathrm{~d}$ after HS
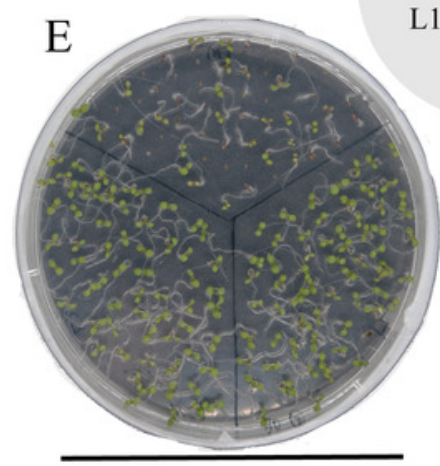

$7 \mathrm{~d}$ after HS

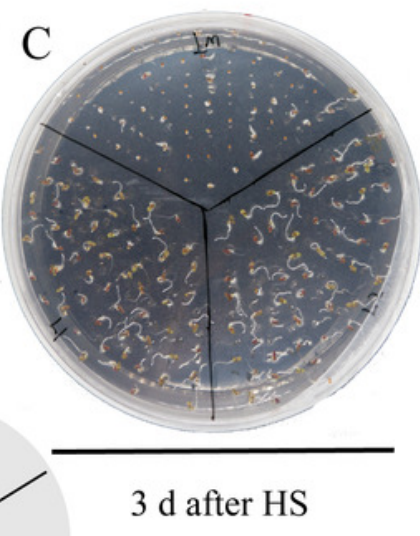

L2

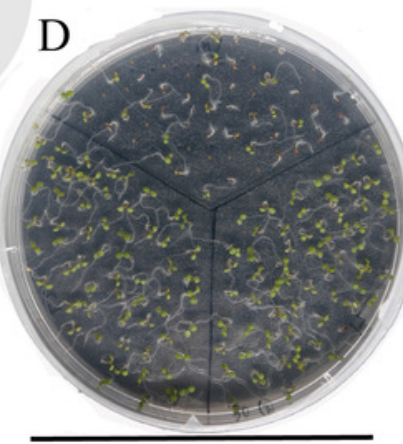

$5 \mathrm{~d}$ after HS 


\section{Table 1 (on next page)}

Basic information of PpHSF gene family members 


\begin{tabular}{|c|c|c|c|c|c|c|c|}
\hline Gene name & Gene ID & $\begin{array}{l}\text { Length of CDS } \\
\text { (bp) }\end{array}$ & $\begin{array}{l}\text { No. of amino acids } \\
\text { (aa) }\end{array}$ & $\begin{array}{c}\text { Molecular } \\
\text { weight }(\mathrm{Da})\end{array}$ & $\begin{array}{l}\text { Predicted } \\
\text { isoelectric } \\
\text { point }(\mathrm{PI})\end{array}$ & $\begin{array}{l}\text { Chromosome } \\
\text { location }\end{array}$ & Subcellular localization \\
\hline PpHSF1 & Prupe.1G021200 & 1068 & 355 & 41166.82 & 4.9 & 1 & Nucleus \\
\hline PpHSF2 & Prupe.1G165500 & 1452 & 483 & 54128.99 & 5.61 & 1 & Nucleus \\
\hline PpHSF3 & Prupe.1G335100 & 1227 & 408 & 46017.53 & 4.81 & 1 & Nucleus \\
\hline PpHSF5 & Prupe.1G433500 & 1170 & 389 & 43557.76 & 7.17 & 1 & Nucleus \\
\hline PpHSF6 & Prupe.2G292100 & 912 & 303 & 33900.98 & 5.19 & 2 & Nucleus \\
\hline PpHSF7 & Prupe.3G108700 & 1314 & 437 & 49855.78 & 5.13 & 3 & Nucleus \\
\hline PpHSF8 & Prupe.4G046000 & 591 & 196 & 22364.46 & 8.75 & 4 & Nucleus \\
\hline PpHSF11 & Prupe.5G031100 & 1551 & 516 & 56261.49 & 4.67 & 5 & Nucleus \\
\hline PpHSF 12 & Prupe.5G093200 & 996 & 331 & 36068.73 & 4.75 & 5 & Nucleus \\
\hline PpHSF13 & Prupe.7G056700 & 735 & 244 & 28001.34 & 5.75 & 7 & Nucleus \\
\hline PpHSF14 & Prupe.7G117200 & 1608 & 535 & 59567.89 & 4.98 & 7 & Nucleus \\
\hline PpHSF 15 & Prupe.7G133600 & 900 & 299 & 33339.83 & 5.07 & 7 & Nucleus \\
\hline PpHSF16 & Prupe.7G206900 & 1458 & 485 & 53632.01 & 5.07 & 7 & Nucleus \\
\hline PpHSF17 & Prupe.7G231100 & 1002 & 333 & 37851.52 & 5.68 & 7 & Nucleus \\
\hline PpHSF18 & Prupe.8G234900 & 1080 & 359 & 40936.56 & 5.58 & 8 & Nucleus \\
\hline
\end{tabular}

1 
Table 2 (on next page)

Cis-elements in the promoters of eighteen PpHSF genes 


\begin{tabular}{|c|c|c|c|c|c|c|c|c|c|c|c|c|c|}
\hline & ABRE & ARE & CGTCA-motif & ERE & MBS & MRE & MYB & MYC & P-box & TGACG-motif & TCA-element & LTR & TGA-element \\
\hline PpHSF1 & 1 & 3 & 2 & 2 & 1 & 1 & 4 & 3 & 2 & 2 & 2 & - & - \\
\hline PpHSF2 & 1 & 2 & 2 & 1 & - & - & 3 & 1 & - & 2 & 1 & 3 & 1 \\
\hline PpHSF3 & 1 & 1 & - & 2 & 1 & 1 & 4 & 2 & - & 1 & - & 1 & 1 \\
\hline PpHSF4 & 3 & 6 & 3 & - & - & - & 3 & - & 1 & 3 & - & 2 & - \\
\hline PpHSF5 & 5 & - & 3 & - & 2 & - & 7 & - & 1 & 3 & 3 & - & - \\
\hline PpHSF6 & 4 & - & 3 & - & 1 & - & 7 & 5 & 2 & 3 & 1 & - & - \\
\hline PpHSF7 & 1 & 5 & - & 1 & - & - & 6 & 3 & - & - & - & $1^{\prime}$ & 1 \\
\hline PpHSF8 & 3 & 3 & 3 & 1 & 1 & 1 & 3 & 8 & 1 & 3 & - & - & 3 \\
\hline PpHSF9 & 5 & 5 & 4 & - & - & 1 & 13 & 4 & - & 4 & - & 2 & 2 \\
\hline PpHSF 10 & 3 & 1 & 5 & 2 & - & - & 2 & 6 & - & 5 & - & - & 2 \\
\hline PpHSF11 & - & 1 & 1 & - & 2 & - & 7 & 8 & - & 1 & - & - & - \\
\hline PpHSF 12 & 6 & - & 1 & 1 & 1 & 1 & 13 & 3 & - & 1 & - & - & 1 \\
\hline PpHSF 13 & 3 & 4 & 1 & - & - & - & 4 & 5 & - & 1 & 1 & 1 & - \\
\hline PpHSF14 & 3 & 4 & 3 & - & 3 & - & 11 & 3 & - & 3 & - & 1 & - \\
\hline PpHSF15 & 8 & 2 & 4 & 1 & 1 & 1 & 6 & 5 & - & 4 & - & - & - \\
\hline
\end{tabular}




\begin{tabular}{|c|c|c|c|c|c|c|c|c|c|c|c|c|c|}
\hline PpHSF16 & 4 & 4 & 1 & 1 & - & 2 & 4 & 11 & - & - & - & - & - \\
\hline PpHSF 17 & 11 & 2 & 2 & 1 & 1 & - & 4 & 6 & - & 2 & - & - & - \\
\hline PpHSF 18 & 13 & 3 & - & 1 & 1 & 2 & 2 & 4 & - & - & - & 1 & - \\
\hline
\end{tabular}

1 